\title{
MEDIDAS DE ESTIMACIÓN DE LA ESTATURA APLICADAS AL ÍNDICE DE MASA CORPORAL (IMC) EN LA EVALUACIÓN DEL ESTADO NUTRICIONAL DE ADULTOS MAYORES
}

\section{STIMATING STATURE MEASUREMENTS APPLIED ON BODY MASS INDEX IN THE NUTRITIONAL ASSESSMENT OF THE ELDERLY}

\author{
Renata Borba de Amorim (1), Maria Auxiliadora Coelho Santa Cruz (1), \\ Paulo Roberto Borges de Souza-Júnior (2), Jurema Corrêa da Mota (2), Catalina González H. (3)
}

(1) Instituto de Nutrição Josué de Castro, Universidade Federal do Rio de Janeiro (UFRJ). Rio de Janeiro, RJ, Brasil.

(2) Centro de Informação Científica e Tecnológica - Fundação Oswaldo Cruz. Rio de Janeiro, RJ, Brasil.

(3) Departamento de Nutrición y Alimentos. Facultad de Farmacia. Universidad de Valparaíso. Valparaíso, Chile.

\begin{abstract}
Introduction: Nutritional assessment in the elderly by anthropometry is part of a toolkit; however, aging causes changes in stature which can alter the interpretation of the measurements. Nutritional assessment is part of this process and stature is a measurement that changes with age. Objective: To analyze the anthropometric measurements of estimating stature on the body mass index to assess nutritional status in the elderly. Method: A cross sectional study with 344 elderly people in the city of Rio de Janeiro, Brazil, was conducted. Anthropometric measurements including body weight $(B W)$, height $(H)$, mid arm span (MAS) and knee height $(\mathrm{KH})$ were carried out and BMI calculated as: BMI1 (BW/H2), BMI2 [BW/(MASx2)2] and BMI3 [BW/(equation of KH)2]. To compare means an independent sample $t$ Student test was used. Chi square and weighted kappa coefficient tests were employed to analyze differences and agreements among the different BMI. Data were analyzed using SPSS 12.0. Results: The BMI1 mean was $24,7 \mathrm{~kg} / \mathrm{m} 2 \pm 5,6 ; \mathrm{BMI} 2$ mean was $23,3 \mathrm{~kg} / \mathrm{m} 2 \pm 6,1$ and BMI3 mean was 24,7 kg/m2 $\pm 5,6$. The weighted kappa between BMI1 and BMI3 was 0,78 and between BMI1 and BMI2 was 0,61. Conclusions: A high association was demonstrated between knee height followed by mid arm span when these measurements were used on BMI. Knee height seems to be the best measurement of real stature.
\end{abstract}

Key words: mid arm span, knee height, assessment, elderly.

Este trabajo fue recibido el 12 de Abril de 2007, aceptado con modificaciones el 7 de Mayo de 2007 y aceptado para ser publicado el 30 de Septiembre de 2008.

\section{INTRODUCCIÓN}

En 1950, había cerca de 204 millones de adultos mayores en el mundo y ya en 1998, este grupo alcanzaba 579 millones de personas, un crecimiento de casi 8 millones por año (1).

La Organización Mundial de la Salud establece que los individuos que viven en países desarrollados inician la etapa de adulto mayor a partir de los 65 años de edad; para los países en vías de desarrollo, en que la esperanza de vida es menor, se considera a partir de los
60 años de edad (2).

Los adultos mayores son reconocidos como grupo de riesgo por todas las alteraciones fisiológicas que ocurren en su organismo, tales como: disminución del metabolismo basal, redistribución de la composición corporal, alteraciones en el funcionamiento del aparato digestivo, modificaciones en la percepción sensorial y en la capacidad masticatoria, disminución de la sensibilidad a la sed, aumento de la frecuencia y gravedad de las enfermedades, en especial, enfermedades crónicas

Artículo basado en la Tesis de Magister: "Mediciones antropométricas de estimación de la estatura en la evaluación del estado nutricional de adultos mayores", 2005, Instituto de Nutrição Josué de Castro, Universidade Federal do Rio de Janeiro (UFRJ). Requisito para la obtención del grado de Magister en Nutrición Humana. 
no transmisibles y efectos secundarios de los fármacos (3-5) que comprometen directa o indirectamente el estado nutricional (6).

La valoración del estado nutricional de este grupo etáreo involucra diversos parámetros como la historia social, evolución clínica, encuesta dietética, indicadores bioquímicos, evaluación antropométrica y de la composición corporal.

De todas las modificaciones corporales que ocurren durante el proceso de envejecimiento, las medidas antropométricas son las más afectadas. En ellas destacan la masa corporal y la estatura. En caso de dificultad en la obtención directa de estas medidas, es posible utilizar métodos de estimación ampliamente descritos en la literatura.

El Índice de Masa Corporal ha sido ampliamente utilizado para la evaluación del estado nutricional de los adultos mayores, sin embargo, por las limitaciones que presenta, debe ir asociado a otros indicadores, observando que el mismo no refleja la composición corporal del individuo. No obstante, debe ser considerado por su fácil aplicación, la gran disponibilidad de datos existentes, la buena relación que tiene con el proceso salud - enfermedad y la inexistencia de otros parámetros mejores (7).

Por la complejidad de la evaluación antropométrica del adulto mayor, es necesario adecuar los métodos e indicadores específicos a este grupo etáreo, utilizándose medidas que garanticen la detección precoz de los problemas nutricionales y el acompañamiento de las intervenciones dietoterapéuticas.

Actualmente, es necesario evaluar los actuales indicadores de valoración del estado nutricional del adulto mayor, con el fin de seleccionar los que más se adecuen a esta población específica, resaltando la importancia de que este grupo poblacional recibe intervenciones en el campo de la nutrición y de la salud pública.

\section{MÉTODOS}

Se realizó un estudio descriptivo de corte transversal, en adultos mayores de ambos sexos institucionalizados en Hogares de Larga Estadía (HLEAM), que tienen convenio con la Secretaría Municipal de Asistencia Social de Rio de Janeiro, RJ, Brasil.

Fueron incluídos en el estudio todos los individuos clasificados como adultos mayores, según la OMS (60 años de edad o más). Se seleccionaron aquellos adultos mayores que presentaron autonomía física (para la evaluación corporal) y mental (para responder la entrevista) y, como requisitos de exclusión se consideró la existencia de deformidades físicas que impidiesen la evaluación antropométrica (desvíos importantes de la columna vertebral o amputación de miembros inferiores o superiores), y la presencia de cifosis importante, ya que esto impide la correcta medición de la estatura.

\section{Ética}

La investigación se inició después de su aprobación por el Comité de Ética en Pesquisa del Hospital Universitario Clementino Fraga Filho - Universidad Federal de Rio de Janeiro. Fue utilizado el protocolo de consentimiento libre e informado de la institución y del adulto mayor.

\section{Antropometría}

El peso, la estatura, la media envergadura de brazo y la altura de rodilla fueron medidos siguiendo técnicas estandarizadas (8 - 11) y la clasificación del estado nutricional por el IMC (Peso/Talla ${ }^{2}$ ), se realizó de acuerdo a los criterios de la OMS para el adulto (1995/1998) $(12,13)$.

La estatura se estimó a través de tres métodos: estatura medida directamente, media envergadura de brazo y altura de rodilla.

La estatura directa se midió usando un antropómetro, con el individuo descalzo, de pie, de espaldas al instrumento, con el peso del cuerpo distribuido en forma pareja sobre ambos pies, los talones y rodillas juntas. Las puntas de los pies separadas levemente en un ángulo de $60^{\circ}$. El dorso estirado y los brazos a los lados, relajados. La cabeza, los omóplatos, las nalgas y los talones en contacto con el plano vertical del instrumento (cuatro puntos de contacto). Se consideró la horizontalidad del plano de Frankfort (línea imaginaria del borde orbitario inferior en el mismo plano horizontal que el conducto auditivo externo). El tope superior del instrumento se hizo descender suavemente aplastando el cabello y haciendo contacto con el vértice del cráneo. Se registró la medida hasta el 0.5 más próximo (14).

La media envergadura de brazo fue medida con el adulto mayor acostado, en posición supina, los brazos abducidos con las palmas de las manos mirando hacia arriba. La medida de media envergadura de brazo corresponde a la extensión del punto en el nivel del segmento central de la incisura yugular del hueso externo hasta el extremo distal de la falange distal del dedo medio derecho, sin considerar la uña. La medida fue tomada con la utilización de una cinta métrica metálica, con precisión de $0,1 \mathrm{~cm}$ y con un ancho máximo de $0,7 \mathrm{~cm}$. La medida de media envergadura de brazo fue tomada 3 veces y se calculó el promedio de estos valores. La estatura, en ambos sexos, equivale al doble del valor encontrado $(9,10)$. 
Estatura $(\mathrm{cm})=$

Media envergadura de brazo x $2(\mathrm{~cm})$

La altura de rodilla se determinó con el adulto mayor acostado en posición supina, con la pierna derecha flexionada, formando con la rodilla un ángulo en $90^{\circ}$, y posicionándose la base del antropómetro de huesos largos debajo del talón del pié y sobre la rótula ajustando hasta presionar la cabeza de la rótula. La lectura del antropómetro fue hecha con el mismo paralelo a toda la extensión del peroné, con una precisión de hasta $1 \mathrm{~mm}$ más próximo, según la recomendación de Chumlea (11). Fueron tomadas dos medidas de altura de rodilla y se calculó el promedio de los valores.

La altura de rodilla se utilizó como una alternativa a la medida de estatura, de acuerdo con la ecuación desarrollada por Chumlea y colaboradores, según sexo y edad, descrita abajo:

\section{Estatura hombres $(\mathrm{cm})=$ \\ [2,02 x AR (cm) $]-[0,04 \times$ edad (años) $]+64,19$ \\ Estatura mujeres $(\mathrm{cm})=$ \\ [1,83 x AR (cm) $]-[0,24 \times$ edad (años) $]+84,88$}

El $\mathrm{IMC}_{1}$, derivado de las medidas de peso corporal y estatura, se calculó con la fórmula Peso/Talla ${ }^{2}$. El $\mathrm{IMC}_{2}$, correspondiente a las medidas de peso corporal y estatura estimada por media envergadura de brazo, se estableció usando $\mathrm{P} /(\mathrm{MEBx} 2)^{2}$. El $\mathrm{IMC}_{3}$, resultante de las medidas de peso corporal y estatura estimada según altura de rodilla, se determinó con P/(ecuación de altura de rodilla) $)^{2}$

\section{Análisis estadístico}

El análisis se realizó por medio de las siguientes medidas de estadística descriptiva: valores mínimo, máximo, promedio y desviación estándar.

Para comparar los promedios se utilizó el test t de Student para muestras independientes y para evaluar las diferencias entre las clasificaciones de IMC fue utilizado, el test $\mathrm{X}^{2}$ se consideraron como significativas las diferencias con valor $\mathrm{p} \leq 0,05$.

Para evaluar el grado de concordancia entre $\mathrm{IMC}_{1}$, $\mathrm{IMC}_{2}$ e $\mathrm{IMC}_{3}$, se usó el coeficiente de Kappa ponderado, con un nivel de significancia de $\mathrm{p}<0,05$.

El análisis de los datos fue realizado a través del software SPSS 12.0 (15).

\section{RESULTADOS}

De las trece unidades geriátricas vinculadas a la SMAS/RJ (Secretaría Municipal de Asistencia Social del Municipio de Rio de Janeiro), fueron intervenidas doce por haberse producido rechazo en una.

Fueron evaluados 344 adultos mayores institucionalizados. De este total, 204 correspondieron a mujeres $(59,3 \%)$, no habiendo deferencias estadísticamente significativas entre los sexos. La edad promedio fue de 75,4 años $( \pm 9,3)$, con un rango que varió de 60 a 117 años de edad.

El análisis estadístico descriptivo, incluyendo valores mínimo y máximo, promedio y desviación estándar de los IMCs $\left(\mathrm{IMC}_{1}, \mathrm{IMC}_{2}\right.$ e $\left.\mathrm{IMC}_{3}\right)$ se muestra en la tabla 1.

El promedio del $\mathrm{IMC}_{1}$ fue de 24,7 (DE $\left.\pm 5,5\right)$. El promedio del $\mathrm{IMC}_{2}$ fue de 23,3 (DE $\pm 6,1$ ), resultando menor al compararlo con el promedio del $\mathrm{IMC}_{1}$, sien-

\section{TABLA 1}

Valores mínimo, máximo, promedio y desviación estándar para $\mathrm{IMC}_{1}$ (masa corporal/estatura), $\mathrm{IMC}_{2}$ (masa corporal/media-envergadura de brazo) e $\mathrm{IMC}_{3}$ (masa corporal/ecuación de la altura de rodilla)

\begin{tabular}{|c|c|c|c|c|c|}
\hline Variables & $\mathbf{N}$ & Mínimo & Máximo & Promedio & $\begin{array}{c}\text { Desviación } \\
\text { estándar }\end{array}$ \\
\hline IMC1 $(\mathrm{kg} / \mathrm{m} 2)$ & 320 & 13,22 & 49,97 & 24,7 & 5,5 \\
\hline IMC2 (Kg./m2) & 275 & 12,60 & 46,36 & $23,3 *$ & 6,1 \\
\hline IMC3 (Kg./m2) & 303 & 14,21 & 48,75 & $24,7 * *$ & 5,6 \\
\hline
\end{tabular}


do esta diferencia estadísticamente significativa ( $\mathrm{p}<$ 0,0001). El promedio del $\mathrm{IMC}_{3}$ fue de 24,7 (DE $\pm 5,6$ ), sin presentar diferencia estadísticamente significativa $(\mathrm{p}=0,855)$.

La tabla 2 presenta la distribución de los adultos mayores por sexo, de acuerdo al diagnóstico nutricional, para las tres metodologías propuestas para el cálculo de estos indicadores ( $\mathrm{IMC}_{1}, \mathrm{IMC}_{2}$ e $\left.\mathrm{IMC}_{3}\right)$.

Al comparar la distribución por estado nutricional para ambos sexos, obtenida por $\mathrm{IMC}_{1}, \mathrm{IMC}_{2}$ e $\mathrm{IMC}_{3}$, se observa, que con el $\mathrm{IMC}_{2}$, aparece un mayor porcentaje de adultos mayores enflaquecidos $(20,1 \%)$ y con estado nutricional normal $(51,5 \%)$, siendo estas diferencias estadísticamente significativas.

La distribución del estado nutricional obtenida por $\mathrm{IMC}_{2}$ presentó una diferencia significativa en comparación con la distribución resultante del $\mathrm{IMC}_{1}(\mathrm{p}<0,001)$, tanto para la muestra total de adultos mayores, como para los de sexo masculino y femenino $(\mathrm{p}<0,001)$.

La distribución de la muestra total de los adultos mayores por estado nutricional diagnosticado con $\mathrm{IMC}_{3}$ no presentó una diferencia significativa al compararla con la distribución derivada del IMC1 $(\mathrm{p}=0,758)$, ni en relación al sexo masculino y femenino $(\mathrm{p}=0,940$ y $\mathrm{p}=0,697$, respectivamente), por lo tanto mostró un patrón de distribución semejante al del $\mathrm{IMC}_{1}$

Al comparar la distribución por estado nutricional y sexo de acuerdo a los tres tipos de IMC, las mujeres presentaron un mayor porcentaje de sobrepeso y obesidad en relación a los hombres. Lo contrario ocurre con la categoría estado nutricional normal, en la que el porcentaje mayor se observa en los hombres.

En la tabla 3, que muestra la distribución de los adultos mayores por grupo etáreo y estado nutricional según los IMCs propuestos, se observa un aumento del porcentaje de enflaquecidos conforme al incremento de la edad en las tres metodologías de cálculo del IMC. Sin embargo, estas diferencias no presentaron significancia estadística: $\mathrm{IMC}_{1}(\mathrm{p}=0,010), \mathrm{IMC}_{2}(\mathrm{p}=0,018)$ e $\mathrm{IMC}_{3}$ $(\mathrm{p}=0,360)$.

El Kappa ponderado entre $\mathrm{IMC}_{1}$ y $\mathrm{IMC}_{2}$ fue de 0,61 , y el Kappa ponderado entre $\mathrm{IMC}_{1} \mathrm{e} \mathrm{IMC}_{3}$ fue de 0,78 , indicando que el IMC cuya estatura fue estimada por la ecuación de la altura de rodilla es más concordante con el

\section{TABLA 2}

Distribución de los adultos mayores por sexo y estado nutricional, según $\mathrm{IMC}_{1}$ (masa corporal/estatura), $\mathrm{IMC}_{2}$ (masa corporal/media-envergadura de brazo) e $\mathrm{IMC}_{3}$ (masa corporal/ecuación de la altura de rodilla)

\begin{tabular}{|c|c|c|c|c|}
\hline Variable & $\begin{array}{l}\text { Diagnóstico } \\
\text { nutricional }\end{array}$ & $\begin{array}{c}\text { Masculino } \\
\%\end{array}$ & $\begin{array}{c}\text { Sexo } \\
\text { Femenino } \\
\%\end{array}$ & $\begin{array}{c}\text { Total } \\
\%\end{array}$ \\
\hline IMC $_{1}$ & $\begin{array}{l}\text { Enflaquecidos } \\
\text { Normales } \\
\text { Sobrepeso } \\
\text { Obesos }\end{array}$ & $\begin{array}{c}10,1 \\
58,1 \\
26,4 \\
5,4\end{array}$ & $\begin{array}{c}9,4 \\
37,7 \\
31,9 \\
20,9\end{array}$ & $\begin{array}{c}9,7 \\
45,9 \\
29,7 \\
14,7\end{array}$ \\
\hline IMC $_{2} *$ & $\begin{array}{l}\text { Enflaquecidos } \\
\text { Normales } \\
\text { Sobrepeso } \\
\text { Obesos } \\
\text { p-valor* }\end{array}$ & $\begin{aligned} & 20,9 \\
& 57,3 \\
& 13,6 \\
& 8,2 \\
&<0,001\end{aligned}$ & $\begin{array}{c}19,5 \\
47,6 \\
17,7 \\
15,2 \\
<0,001\end{array}$ & $\begin{array}{c}20,1 \\
51,5 \\
16,1 \\
12,4 \\
<0,001\end{array}$ \\
\hline $\mathrm{IMC}_{3}$ & $\begin{array}{l}\text { Enflaquecidos } \\
\text { Normales } \\
\text { Sobrepeso } \\
\text { Obesos } \\
\text { p-valor** }\end{array}$ & $\begin{array}{c}9,5 \\
56,0 \\
28,4 \\
6,0 \\
p=0,940\end{array}$ & $\begin{array}{c}9,8 \\
38,3 \\
34,4 \\
17,5 \\
p=0,697\end{array}$ & $\begin{array}{c}9,7 \\
45,2 \\
32,1 \\
13,0 \\
p=0,758\end{array}$ \\
\hline
\end{tabular}


IMC en el cual se utilizó la estatura directamente medida, seguido del IMC calculado a través de la estimación de la estatura por la media envergadura de brazo.

\section{DISCUSIÓN}

La utilización de la media - envergadura de brazo como medida de estimación de la estatura subestimó el promedio de IMC, mientras que la altura de rodilla no mostró una diferencia estadísticamente significativa en el indicador.

El estudio de Lera et al (16), a través de la alta concordancia encontrada en estudios americanos de estatura, corrobora que el largo de la altura de rodilla se mantiene estable durante la vida adulta, razón por la cual es una de las alternativas para la predicción de la estatura.

Al comparar el $\mathrm{IMC}_{2}$ y el IMC $\mathrm{IMC}_{3}$ con el IMC $\mathrm{IM}_{1}$, se encontró que el IMC en que se utilizó la media envergadura de brazo mostró mayor prevalencia de enflaquecimiento y normalidad, y el IMC basado en el uso de la altura de rodilla identificó mayor prevalencia de sobrepeso. En un estudio similar de Garcia (17), en que se usó la envergadura de brazo, se encontró mayores porcentajes de enflaquecimiento y normalidad y menores porcentajes de sobrepeso y obesidad.

En este trabajo se observó, en el adulto mayor, una relación directa entre la edad cronológica y la prevalencia de enflaquecimiento. Esto está ampliamente postulado en la literatura, como en el estudio de Perissinotto et al (18), realizado en individuos mayores de 65 años de edad, cuyos resultados mostraron una disminución estadísticamente significativa de la masa corporal y estatura con el incremento de la edad.

Las mujeres investigadas en este estudio presentaron una mayor prevalencia de sobrepeso y obesidad en comparación con los hombres. El estudio de Lasheras et al (19) encontró promedios de IMC para ambos sexos superiores a $25 \mathrm{~kg} / \mathrm{m}^{2}$, en cuanto Perissinotto et al, identificaron que el IMC fue significativamente mayor $\left(27,6 \mathrm{~kg} / \mathrm{m}^{2}\right)$ en las mujeres en relación a los hombres $\left(26,4 \mathrm{~kg} / \mathrm{m}^{2}\right)$. En el mismo estudio, la obesidad estuvo presente en el $28 \%$ de las mujeres y el $16 \%$ de los hombres, lo que probablemente corrobora los hallazgos del presente trabajo, donde fue identificado un $31,9 \%$ de sobrepeso y un $20,9 \%$ de obesidad para el sexo femenino y un $26,4 \%$ de sobrepeso y un $5,4 \%$ de obesidad para el sexo masculino.

\section{TABLA 3}

Distribución de los adultos mayores, por grupo atareo y estado nutricional, según $\mathrm{IMC}_{1}$ (masa corporal/estatura), $\mathrm{IMC}_{2}$ (masa corporal/media-envergadura de brazo) e $\mathrm{IMC}_{3}$ (masa corporal/ecuación de la altura de rodilla).

\begin{tabular}{|c|c|c|c|c|}
\hline \multirow[t]{2}{*}{ Variable } & \multirow{2}{*}{$\begin{array}{l}\text { Diagnóstico } \\
\text { Nutricional }\end{array}$} & \multicolumn{3}{|c|}{ Grupo etareo (años) } \\
\hline & & $\begin{array}{c}60-69 \\
\%\end{array}$ & $\begin{array}{c}70-79 \\
\%\end{array}$ & $\begin{array}{c}\geq 80 \\
\%\end{array}$ \\
\hline \multirow{4}{*}{$\mathrm{IMC}_{1} *$} & Enflaquecidos & 2 & 9,6 & 16,2 \\
\hline & Normales & 55,6 & 38,5 & 44,4 \\
\hline & Sobrepeso & 28,3 & 33,7 & 27,4 \\
\hline & Obesos & 14,1 & 18,3 & 12,0 \\
\hline \multirow{4}{*}{$\mathrm{IMC}_{2} * *$} & Enflaquecidos & 10,3 & 18,5 & 28,8 \\
\hline & Normales & 51,3 & 53,3 & 50,0 \\
\hline & Sobrepeso & 19,2 & 19,6 & 10,6 \\
\hline & Obesos & 19,2 & 8,7 & 10,6 \\
\hline \multirow{4}{*}{$\mathrm{IMC}_{3} * * *$} & Enflaquecidos & 4,3 & 10,6 & 13,4 \\
\hline & Normales & 51,6 & 40,4 & 43,8 \\
\hline & Sobrepeso & 31,2 & 36,2 & 29,5 \\
\hline & Obesos & 12,8 & 13,4 & \\
\hline \multicolumn{5}{|c|}{$\begin{array}{l}* \mathrm{p}=0,010 \text {; Test X2: comparación entre IMC1 vs. Grupo etareo } \\
* * \mathrm{p}=0,018 ; \text { Test X2: comparación IMC2 vs. Grupo etareo }\end{array}$} \\
\hline$* * \mathrm{p}=0,360$ & nparación IMC3 vs. & & & \\
\hline
\end{tabular}


La American Academy of Family Physicians (20) propone para los adultos mayores otra clasificación de los puntos de corte de IMC, diferente de la establecida para adultos por la OMS (1995/1998). Recomienda que en el adulto mayor sobre 65 años se considere un IMC entre 24 y $29 \mathrm{~kg} / \mathrm{m}^{2}$ y que valores bajo $22 \mathrm{~kg} / \mathrm{m}^{2}$ indiquen enflaquecimiento. Sin embargo, su utilización aún no es ampliamente recomendada por la falta de estudios de validación en otras poblaciones (21).

Otros autores proponen valores de referencia diversos, como Kuczmarski et al (22) que determinaron valores de normalidad entre 22,4 y $27,1 \mathrm{~kg} / \mathrm{m}^{2}$ para hombres y de 21,7 a $28,4 \mathrm{~kg} / \mathrm{m}^{2}$ para mujeres, encuanto Perissinotto et al, propone de 23 a $27,4 \mathrm{~kg} / \mathrm{m}^{2}$ para hombres y de 23,5 a $30,1 \mathrm{~kg} / \mathrm{m}^{2}$ para mujeres, lo que corresponden al $\mathrm{P}_{25}$ y $\mathrm{P}_{75}$, respectivamente. Yá Lebrão (23), en el Proyecto SABE, del Município de São Paulo, muestra valores de 23 a $28 \mathrm{~kg} / \mathrm{m}^{2}$ para ambos sexos.

La posible influencia de la edad en los puntos de corte es discutida por la OMS (1995), que muestra que los valores del IMC de adultos pueden ser utilizados para los adultos mayores de 60-69 años, sin embargo aún no está bien establecido si estos son adecuados para los adultos mayores arriba de 70 años.

Usar los mismos puntos de corte para todos los grupos etários puede significar que no todos los adultos mayores en riesgo nutricional, sean detectados. Otro problema es que los puntos de corte disponibles han sido utilizados para identificar solamente la presencia de alteraciones nutricionales ya instaladas. Considerando que el objetivo de una evaluación efectiva del estado nutricional en el adulto mayor y de cualquier grupo etáreo, no es solamente detectar la alteración nutricional, sino anticiparla y prevenir su inicio o identificarla antes que alcance importancia clínica (24).

De esta forma, es cuestionable el valor del punto de corte propuesto por la OMS (1995/1998) para un diagnóstico del estado nutricional en el adulto mayor, una vez que la masa corporal asociada a menor mortalidad aumenta con la edad (25) y el riesgo relativo de mortalidad asociado a un alto IMC disminuye con la edad (26).

Otro asunto relevante es si los puntos de corte del IMC propios para los adultos mayores deben ser clasificados de acuerdo con el sexo, teniendo en cuenta las diferencias en las tendencias de modificación de la composición corporal entre hombres y mujeres que se producen en este grupo etáreo (18).

Se conoce que tanto la masa corporal como la estatura en el adulto mayor sufren alteraciones de acuerdo al grupo etáreo. Idealmente podrían ser desarrolladas curvas de referencia nacionales e internacionales de
IMC por edad, teniendo en vista el comportamiento variable de las dos medidas utilizadas en el indicador y las características propias de la población. Otro cuidado en los estudios del tema del envejecimiento, es que se debe tener cautela con los análisis en los grupos de edad más avanzada por causa de los posibles efectos de confusión resultantes de la tendencia secular, la mortalidad y la sobrevivencia selectiva (27).

Las diferencias en las medidas de estimación de la estatura por las medidas de media envergadura de brazo y altura de rodilla, al ser aplicadas en el IMC, pueden alterar el diagnóstico nutricional individual. El estudio de Kirk et al (28), mostró que la gran diferencia encontrada entre las medidas (mayores de $10 \mathrm{~cm}$ ), determina un problema en la práctica clínica, al ser aplicadas al IMC, a pesar de la literatura científica sugerir su utilización como alternativas confiables de estimación de la estatura. Sin embargo, ante los elevados índices de concordancia identificados en este estudio, parecen poder ser usadas en el nivel poblacional con relativa seguridad.

\section{CONCLUSIÓN}

Los resultados arrojan que es posible utilizar la media envergadura de brazo y la altura de rodilla como medidas de estimación de la estatura o el Índice de Masa Corporal en el adulto mayor, debido a la alta concordancia encontrada entre los IMCs en que estas fueron usadas como medidas de estimación de la estatura.

Teniendo en cuenta los resultados encontrados en este estudio y en la literatura, la altura de rodilla se mostró como la medida que más se aproxima al valor real de la estatura, para ser utilizada en la ecuación del Índice de Masa Corporal, en la evaluación del estado nutricional de adultos mayores,

\section{RESUMEN}

Introducción: En el adulto mayor la antropometría es parte de un conjunto de herramientas para la evaluación nutricional, sin embargo la estatura se modifica con el envejecimiento, lo que puede alterar la interpretación de los resultados. Objetivo: Analizar las medidas de estimación de la estatura aplicadas al Índice de Masa Corporal (IMC) para la evaluación nutricional de adultos mayores. Método: Estudio transversal con 344 adultos mayores de la Municipalidad de Rio de Janeiro. Se midió el peso $(\mathrm{P})$, la estatura $(\mathrm{E})$, la media-envergadura de brazo (MEB) y la altura de rodilla (AR) y se calculó Índice de Masa Corporal(IMC) para: $\mathrm{IMC}_{1}\left(\mathrm{Peso}_{\mathrm{Talla}^{2}}\right), \mathrm{IMC}_{2}[\mathrm{P} /$ $\left.(\mathrm{MEBx} 2)^{2}\right]$ e $\mathrm{IMC}_{3}\left[\mathrm{P} /(\text { ecuación de altura de rodilla })^{2}\right]$. En la comparación de los promedios se utilizó el test $t$ de Student para muestras independientes y las diferencias y concordancia entre IMCs fueron analizados con test 
$\mathrm{X}^{2}$ y el Coeficiente Kappa ponderado, respectivamente. Resultados: El promedio de $\mathrm{IMC}_{1}$ fue $24,7 \mathrm{~kg} / \mathrm{m}^{2} \pm 5,6$, $\mathrm{IMC}_{2} 23,3 \mathrm{~kg} / \mathrm{m}^{2} \pm 6,1$ e $\mathrm{IMC}_{3} 24,7 \mathrm{~kg} / \mathrm{m}^{2} \pm 5,6$. El Kappa ponderado entre $\mathrm{IMC}_{1}$ e IMC $\mathrm{IMe}_{3}$ fu,78 y entre $\mathrm{IMC}_{1} \mathrm{e}$ $\mathrm{IMC}_{2}$ 0,61. Conclusiones: El IMC basado en la altura de rodilla presentó una fuerte asociación con el IMC basado en la estatura medida directamente, seguido del IMC en que fue usada la media-envergadura de brazo. La altura de rodilla sería la medida más próxima al valor real de la estatura.

Palabras claves: media envergadura, altura de rodilla, evaluación, adulto mayor.

Dirigir la correspondencia a:

Profesora

Renata Borba de Amorim

Universidade Federal do Rio de Janeiro

Instituto de Nutrição Josué de Castro

Departamento de Nutrição Social e Aplicada

Avenida Brigadeiro Trompowisky, s/n

CCS - Bloco J2

21941590 - Rio de Janeiro, RJ - Brasil

Tel.: (5521) 2562-6595

Fax.: (5521) 2280-8343

e-mail: amorimrb@yahoo.com.br

\section{BIBLIOGRAFÍA}

1. Andrews GA. Los desafíos del proceso de envejecimiento en las sociedades de hoy y del futuro. In: Encuentro Latinoamericano y del Caribe - Sobre Las Personas de Edad. CELADE, (Santiago - Chile), Seminarios y Conferencias - CEPAL, 2, 2000 pp. $247-256$

2. Novelo de López H. Aspectos demográficos del adulto mayor. Nutr Clin 2003; 6 (1): 63-69.

3. Quitero-Molina R. Nutrición en los ancianos. Geriatrika 1993; 9(1):14 - 18.

4. Nogués R. Factores que afectan la ingesta de nutrientes en el anciano y que condicionan su correcta nutrición. Nutr Clin 1995; 15(2): 39-44.

5. Campos M, Monteiro J, Ornelas A. Fatores que afetam o consumo alimentar e a nutrição do idoso. Rev Nutr Campinas 2000; 13(3): 157-165.

6. Kendrick Z, Lowenthal D. Metabolical and nutritional considerations for exercising older adults. Geriatrics 1994; 20 (10): 558-568.

7. Najas M. Avaliação do estado nutricional de idosos a partir da utilização da medida do comprimento da perna - "knee height"- como método preditor da estatura. São Paulo, 1995. [Tese - Mestrado - Universidade Federal de São Paulo - Escola Paulista de Medicina].
8. Gibson R. Principles of nutritional assessment. New York, Oxford University Press, 1990, pp. 691.

9. Lohman T, Roche A, Martorell R. Anthropometric standardization reference manual. Champaign, IL: Human Kinetics Books, 1988.

10. Ismail S, Manadhar M. Better nutrition for older people: Assessment and Action. London School of Hygiene and Tropical Medicine, 1999, pp 80.

11. Chumlea W, Roche A, Steinbaugh M. Estimating stature from knee height for persons 60-90 years of age. J Am Geriatr Soc 1985; 33: 116-120.

12. OMS - Organização Mundial de Saúde. Physical Status: The use and interpretation of anthropometry. Geneve: World Health Organization, 1995.

13. OMS - Organização Mundial de Saúde. Obesity: Preventing and managing the global epidemic. Report of a WHO consultation on obesity. Geneva, 1998.

14. Gordon C, Chumlea W, Roche A. Stature, recumbent length, and weight. In: Lohman T, Roche A, Martorell R. Anthropometric standardization reference manual. Abridged Edition 1991: 3-8.

15. SPSS - Statistical Package for the Social Sciences [Windows]. V. 12.0.

16. Lera L, Santos J, García C, Arroyo P, Albala C. Predictive equations for stature in the elderly: a study in three Latin American cities. Annals of Human Biology 2005; 32(6): 773-781.

17. Garcia A. Indicadores antropométricos na avaliação nutricional de idosos institucionalizados. 119f. 2000. (Dissertação de Mestrado em Nutrição) Centro de Ciências da Saúde, Universidade Federal de Pernambuco, Recife, 2000.

18. Perissinotto E, Pisent C, Sergi G, Grigoletto F, Enzi G. Anthropometric measurements in the elderly: age and gender differences. Br J Nutr 2002; 87: 177-186.

19. Lascheras C, González C, García A, Patterson A, Fernádez S. Dietary intake and biochemical indicators of nutritional status in an elderly institutionalized and non- institutionalized population. Nutr Res 1999; 19(9): 1299- 312.

20. American Academy of Family Physicians. The American Dietetic Association. National Council on the Aging Inc. Incorporating Nutrition Screening and Interventions into Medical Practice: A monograph for physicians. 1997.

21. Anjos L. Índice de Massa Corporal (massa corporal. estatura-2) como indicador do estado nutricional de adultos: revisão da literatura. Rev Saúde Pública 1992; 26(6): 431-6.

22. Kuczmarski M, Kuczmarski R, Najjar M. Descrip- 
tive anthropometric reference data for older Americans. J Am Diet Assoc 2000; 100(1): 59-66.

23. Lebrão M. O projeto SABE em São Paulo: uma visão panorâmica. In: Lebrão, M; Duarte, YAO (Orgs.). SABE: Saúde, Bem-estar e Envelhecimento. O Projeto SABE no Município de São Paulo: uma abordagem inicial. Brasília: Organização PanAmericana de Saúde; 2003: pp. 35-43.

24. Beck A, Ovesen L. At which body mass index and degree of weight loss should hospitalized elderly patients be considered at nutritional risk? Clin Nutr 1998; 17(5): 195-198.

25. Allison M, Keller C. Physical activity in the elderly: benefits and intervention strategies. Nurs Pract 1997; 22(8): 53-4.

26. Stevens J, Cai J, Pamuk E, Williamson D, Thun M. The effect of age on the association between bodymass index and mortality. NEJM 1998;338(1):1-7.

27. Tavares E, Anjos L. Perfil antropométrico da população idosa brasileira. Resultados da Pesquisa Nacional sobre saúde e nutrição. Cad Saúde Pública 1999;15(4): 759-768.

28. Kirk S, Hawke T, Sandford S, Wilks Z, Lawrenson $\mathrm{S}$. Are the measures used to calculate BMI accurate and valid for the use in older people? J Hum Nutr Diet 2003; 16: 365-370. 